\title{
Synthesis of exotic nuclei in heavy-ion collisions at higher energies
}

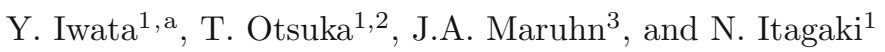 \\ 1 Department of Physics, University of Tokyo, Hongo 7-3-1, Tokyo 113-0033, Japan \\ 2 Center for Nuclear Studies, University of Tokyo, Hongo 7-3-1, Tokyo 113-0033, Japan \\ 3 Institut für Theoretische Physik, Universität Frankfurt, 60325 Frankfurt, Germany \\ Original article: Eur. Phys. J. A 42, 613 (2009) DOI: 10.1140/epja/i2009-10851-1
}

Received: 14 September 2009

Published online: 17 September 2009 - (C) Società Italiana di Fisica / Springer-Verlag 2009

Due to a calculation mistake during writing the manuscript, in sect. 2.1, the estimated value of the upper limit using the standard value of $k_{F}$ is not correct. The text should read as follows:

"By taking the Fermi momentum as the standard constant $k_{F}=1.36 \mathrm{fm}^{-1}$, we have $38.4 A_{1} A_{2} /\left(A_{1}+A_{2}\right)^{2} \mathrm{MeV} / \mathrm{A}$ for the upper energy-limit.... For instance, when we take symmetric cases with $A_{1}=A_{2}$, the standard value for the upper energy-limit of charge equilibration is calculated to be $9.6 \mathrm{MeV} / \mathrm{A} . "$

This change is not substantial and does not affect the conclusions presented in this work.

\footnotetext{
${ }^{a}$ e-mail: iwata@tkyntm.phys.s.u-tokyo.ac.jp
} 